\title{
OPERATIONAL WEAR AND FRICTION IN MEMS DEVICES
}

\author{
G. Subhash $^{1,2}$, A.D. Corwin ${ }^{2}$ and M. P. de Boer ${ }^{2}$ \\ ${ }^{1}$ Michigan Technological University, Houghton, MI 49931 \\ ${ }^{2}$ Sandia National Laboratories, Albuquerque, NM 87185
}

\begin{abstract}
A polycrystalline silicon (polysilicon) surface-micromachined 'Nanotractor' device was used to investigate the evolution sequence of wear mechanisms and coefficient of friction (COF) in MEMS structures. This friction based stepped motor was operated until failure under well controlled test conditions to induce accelerated wear. Static coefficient of friction was measured intermittently at selected intervals during wear cycling. The COF increased by more than an order magnitude from its virgin value (0.2) during the wear cycling and then decreased gradually to reach a steady state value around 0.45 . The travel distance over a prescribed fixed number of steps was monitored and was found to decrease gradually with an increased number of wear cycles. These devices operated for around 200,000 cycles. It was found that the intermittent friction tests also contributed to rapid wear on the surfaces and decreased operational life. By minimizing the number of friction tests performed during the wear cycling, the operational life of the device was extended well beyond 700,000 cycles. Extensive microscopic observations revealed that the wear process transitions from summit plasticity to flattening of fine debris to debris agglomeration to third body wear and finally to localized severe wear. The failure mechanism is identified as locking of the contact surfaces in the grooves created due to severe wear. The current studies can be useful in developing reliability guidelines and test procedures for estimation of residual life of MEMS devices.
\end{abstract}

\section{INTRODUCTION}

Recent advances in design and development of MEMS devices with contact or rubbing surfaces have lead to increased interest in their operational reliability. In particular, the tribological behavior of such devices has been the focus of such investigations. Although numerous investigations have been reported on the wear and frictional properties of silicon based materials [1,2], the reports on wear behavior of MEMS devices are limited [3-5]. Investigations on actual devices are essential for establishing operational limits and for identification of required design modifications for improved performance.

\section{DEVICE DESCRIPTION}

The nanotractor is a friction based MEMS device made of surface machined polysilicon material. It was fabricated using SUMMIT VTM technology at Sandia National Laboratories. It consists of five major components as shown in Fig.1: a leading clamp, a trailing clamp, a central actuation plate connecting the two clamps, a set of four linear guiding springs and a non-linear spring that applies force opposing the motion of the device clamps. A scanning electron micrograph of the device is shown in Fig.1. Its design details and operational principle have been described by de Boer et al. [6]. The device operates on electrostatic actuation principles through the application of phased sequence of voltage signals on the leading clamp, the actuation plate and the trailing clamp successively. Upon application of voltage the friction pads beneath each clamp come in contact with their lower counterface (wear track) to provide frictional resistance. The actuation of the central plate causes its out of plane deflections to be converted to in-plane displacement of the clamps and results in motion similar to an 'inchworm' in nature. The step size per actuation cycle is around $50 \mathrm{~nm}$. The device is walked by prescribing 200 steps to a travel distance of around 10 microns.

\section{TEST PROCEDURE}

The device can be traversed back and forth (defined as one cycle) by prescribing the number of steps. To accelerate the wear between the contact surfaces, the device is operated with an additional normal load of around $100 \mu \mathrm{N}$ on the leading clamp during its motion. At selected intervals of wear cycling, static coefficient of friction was measured by conducting a friction test, where the device is walked to its farthest position against the load spring and held in place with a large normal force on the leading clamp. The normal force is gradually decreased until the device starts to slide back towards its zero position due to the 
tangential force (which is related to the distance) exerted by load spring. Based on the normal force at which the device starts to move and the tangential force, friction coefficient can be determined.

\section{TEST RESULTS}

A typical test result showing the travel distance as a function of number of wear cycles is presented in Fig.2. The travel distance decreases gradually until device failure (unable to move any longer). The coefficient of friction measured during this test is shown in Fig. 3. The friction values increase gradually from 0.2 to 3 and then decrease back to a steady value of around 0.45 . Careful observation of the features (noise like) in Fig.2 reveals periodic drops in the travel distance. These intervals correspond to the cycles immediately after the friction tests. Thus the friction test also appears to strongly influence the evolving surface conditions. Thus by minimizing the number of friction tests the device life has been extended to almost 700,000 cycles.

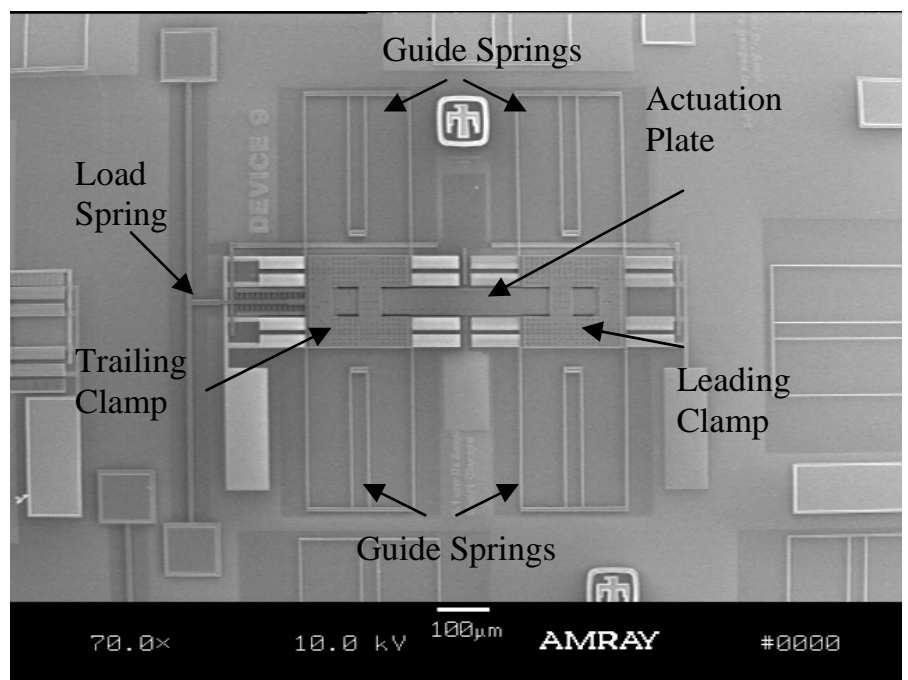

Figure 1: Scanning electron micrograph of nanotractor

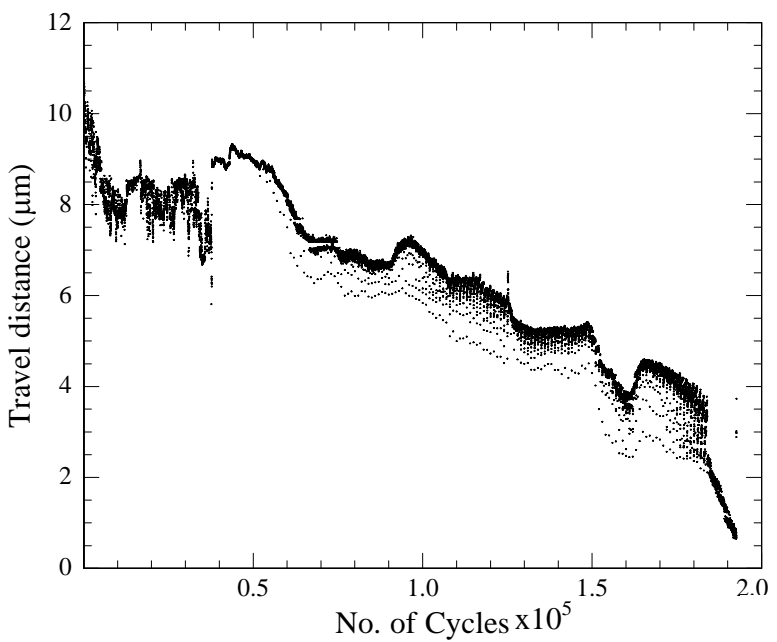

Figure 2: Travel distance in each cycle as a function of wear cycles 


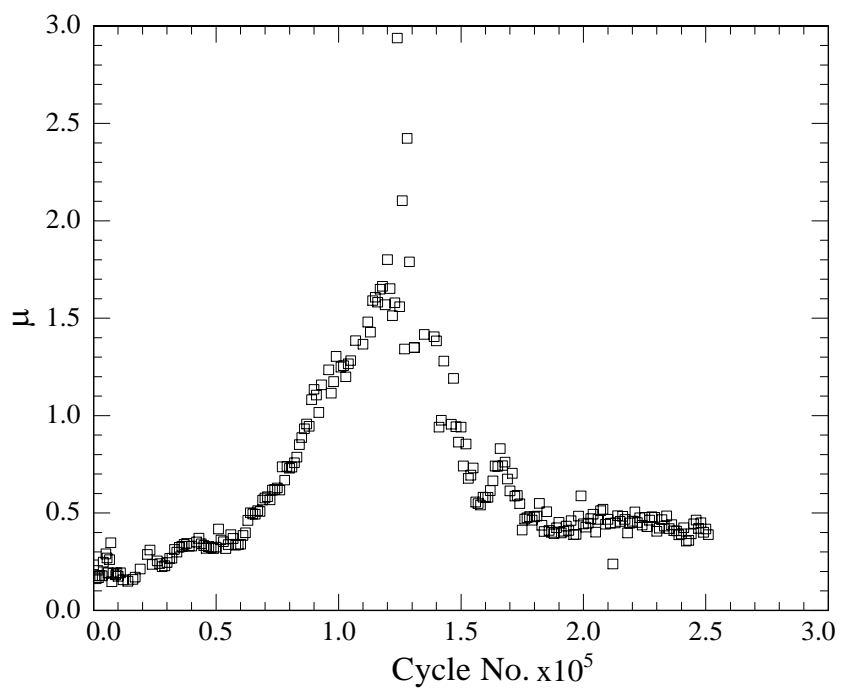

Figure 3: Evolution of friction coefficient as a function of wear cycles

\section{WEAR CHARACTERIZATION}

The friction pad and the wear track were observed under a scanning electron microscope to determine the mechanisms of wear. Fig. 4 illustrates various phases of material removal during wear testing. Fig.4(a) reveals early stages of wear in terms of asperity blunting, plasticity associated with the crushed wear debris and formation of a thin film on the surface. The increase in friction coefficient shown earlier is attributed to the increased adhesion between the contact surfaces due to this film and subsequent increase in contact area. As wear cycling progresses, this wear debris agglomerates and initiates third body wear evidenced by the scratches shown in Fig.4(b). Finally, in Fig.4(c), severe wear in one location on the friction pad, which ultimately causes device failure, due to locking of the counter face in this grove.

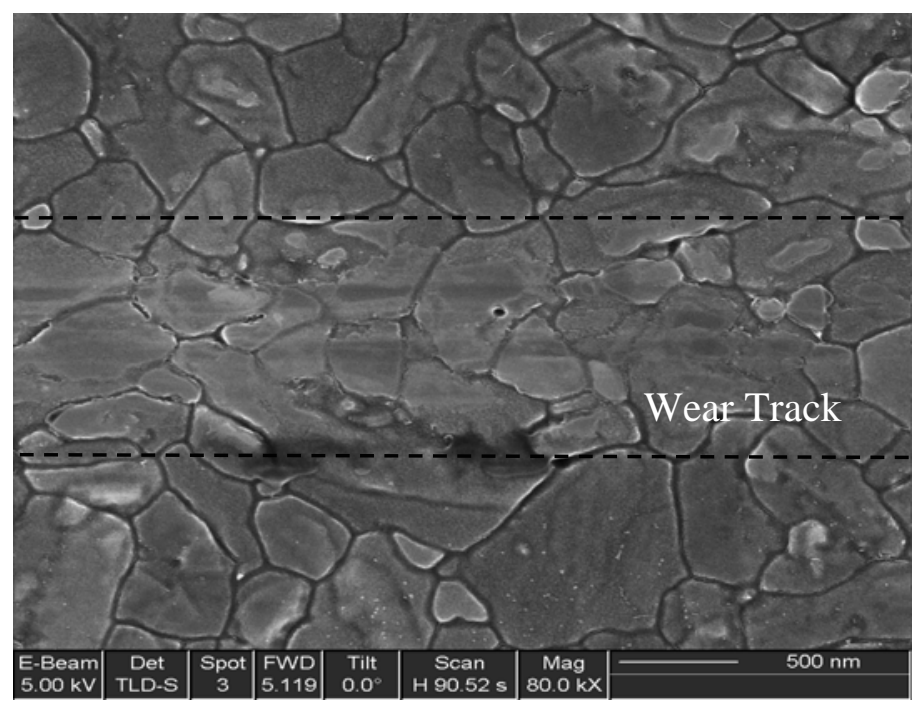

Figure 4(a): Plastically deformed wear debris forming a thin film on the lower counterface 


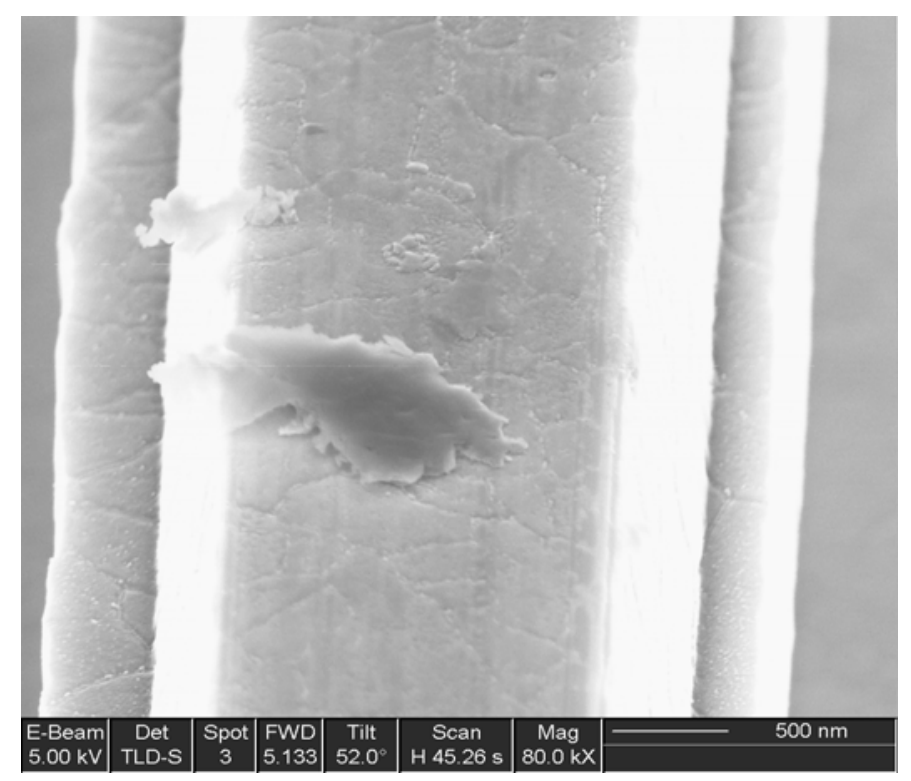

Figure 4(b): Wear debris agglomeration and scratches on the upper counterface

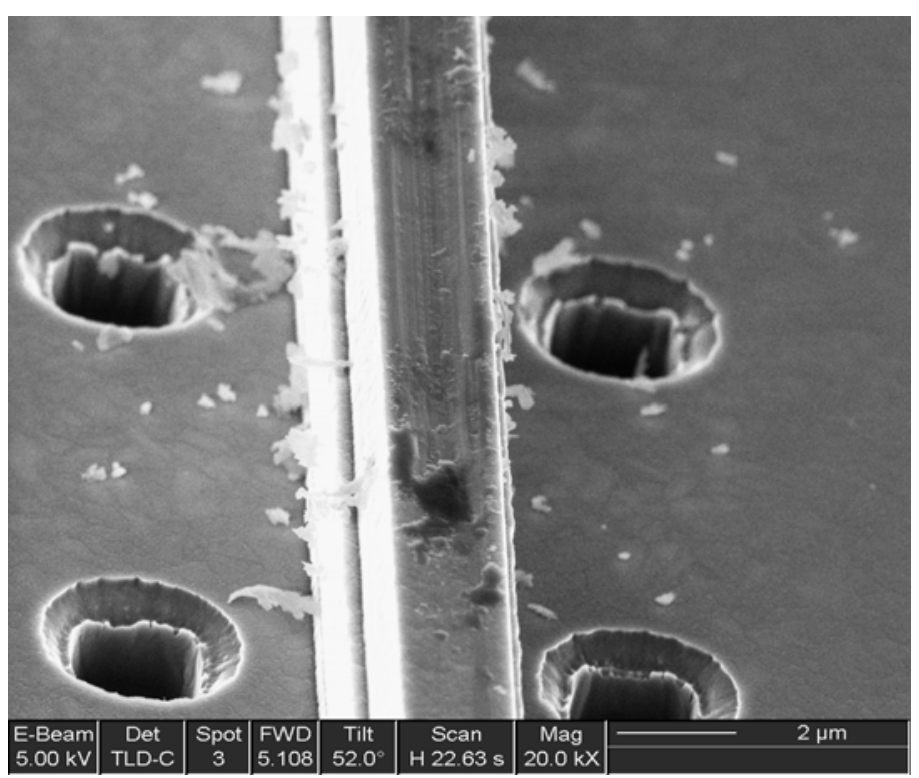

Figure 4(c): Severe wear on the upper counterface

6 CONCLUSIONS

This study identifies various phases in the tribological behavior of the polysilicon surfaces. The wear process transitions from summit plasticity to flattening of fine debris to debris agglomeration to third body wear and finally to localized severe wear. Surprisingly, the measured friction coefficient values can not be used to monitor the incipient failure of the device. Failure in this device occurs not due to fatigue or mechanical failure but solely due to wear phenomena. 


\section{ACKNOWLEDGMENTS}

${ }^{1}$ This work was accomplished while on sabbatical leave at Sandia National Laboratories. Sandia is a multiprogram laboratory operated by Sandia Corporation, a Lockheed Martin Company, for the US Department of Energy's National Nuclear Security Administration under contract No. DE-AC0494AL85000.

\section{REFERENCES}

[1] Bhushan, B. and X.D. Li, Micromechanical and tribological characterization of doped single-crystal silicon and polysilicon films for microelectromechanical systems devices, Journal of Materials Research 12 (1997) 54-63

[2] Beerschwinger, U., T. Albrecht, D. Mathieson, R.L. Reuben, S.J. Yang, and M. Taghizadeh, Wear at Microscopic Scales and Light Loads for MEMS Applications, Wear 181 (1995) 426-435.

[3] Tanner, D.M. and M.T. Dugger, Wear mechanisms in reliability methodology, Proceedings of the SPIE: The International Society for Optical Engineering 4980 (2003) 22-40

[4] Mehregany, M., S.D. Senturia, and J.H. Lang, Measurement of wear in polysilicon micromotors, IEEE Transactions on Electron Devices 39 (1992) 1136-1143.

[5] Corwin, A.D. and M.P. de Boer, Effect of adhesion on dynamic and static friction in surface micromachining, Applied Physics Letters, Vol.84, No.13, 2451-2453 (2004).

[6] de Boer, M.P., David L. Luck, W.R. Ashurst, R.Maboudian, A.D. Corwin, J.A. Walraven and J. M. Redmond, "High performance surface-micromachined inchworm actuator, Journal of Microelectromechanical Systems, Vol. 13, No.1, 63-74 (2004). 